\title{
Solution of Economic Load Dispatch Problem with Smooth and Non-Smooth Fuel Cost Functions Including Line Losses Using Genetic Algorithm
}

\author{
Attia A. El-Fergany, Member, IACSIT
}

\begin{abstract}
The paper presents an application of Genetic Algorithm (GA) to solve Economic Load Dispatch (ELD) problems with smooth and non-smooth fuel cost objective functions. Main objective of ELD is to determine the most economic generating dispatch required to satisfy the predicted load demands including line losses over a certain period of time while relaxing various equality and inequality constraints. The unit Min/Max operational constraints, effects of valve-point loading ripples and line losses are considered for the practical applications. Several cases were tested and verified, among of them, two cases of 6-units and 40-units systems including losses with smooth and non-smooth cost functions were tested, verified and compared with previous reported researches. Finally, it can be concluded that GA proves an excellent viability to optimize and solve problems of ELD. Numerical simulations indicate an improvement in total fuel cost savings.
\end{abstract}

Index Terms-Economic load dispatch problem, Genetic Algorithm (GA), Smooth and non-smooth cost functions.

\section{INTRODUCTION}

ELD is an optimization problem and may be solved by known means of numerical optimization. ELD is the short-term determination of the optimal output of a number of electricity generation facilities, to meet the system demand, at the lowest possible fuel cost, while serving power to the public in a robust and reliable manner [1-3]. Performing an ELD more frequently (e.g., 5 or 15 minutes rather than each hour) affects the level of costs.

Many recent works have been around Artificial Intelligence (AI) methods, on par with the development of AI optimization theories, such as Artificial Neural Networks (ANN), Simulated Annealing (SA), Genetic Algorithms (GA), Differential Evolution (DE), Particle Swarm Optimization (PSO), Evolutionary Programming (EP), and hybrid methods [3-6]. ELD algorithms for thermal unit system involving combined cycle units presented in [7]. Online solving of economic dispatch problem using neural network approach and comparing it with classical methods were presented in [8]. The Evolutionary Algorithms (EAs) are different from the conventional optimization methods, and they do not need to differentiate cost function and constraints. Theoretically, like SA, EAs converge to the global optimum solution. EAs, including Evolutionary Programming (EP), Evolutionary Strategy (ES), and GA are AI methods for optimization based on the mechanics of

Manuscript received on June 3, 2011; revised on July 5, 2011.

A. El-Fergany is with Dept. of Electrical Power Engineering, Faculty of Engineering, Zagazig University- Zagazig, Egypt. (e-mail: el_fergany@ yahoo.com) natural selection, such as mutation, recombination, reproduction, crossover, selection, etc [9-11]. Many researchers exerted lot of work to improve many optimization and intelligent techniques to solve ELD problems such as PSO [12-16], GA [16-17, 23], Hopfield solution [19] and SA [18].

In this paper, GA is proposed as a methodology for ELD with convex and non-convex cost functions with taking effects of valve-point loading into consideration. The data of 6 generating units and 40 generating units have taken to which are numerical tested and compared. The results are compared with [20-22].

\section{ELD PROBLEM FORMULATION}

The primary concern of an ELD problem is the minimization of its objective function. The total cost generated that meets the demand and satisfies all other constraints associated is selected as the objective function [1, 3]. In general, the ELD problem can be formulated mathematically as a constrained optimization problem with an objective function of the form, as illustrated in (1): Objective Function:

$$
\text { Minimize: } \mathrm{FC}_{\mathrm{T}}=\sum_{\mathrm{i}=1}^{\mathrm{N}} \mathrm{FC}_{\mathrm{i}}\left(\mathrm{P}_{\mathrm{i}}\right)
$$

where $\mathrm{FC}_{\mathrm{T}}$ is the total generation cost; $\mathrm{N}$ is the total number of generating units; $\mathrm{FC}_{\mathrm{i}}$ is the power generation cost function of the $i^{\text {th }}$ unit.

\section{A. Classical Smooth Fuel Cost Functions}

Generally, the fuel cost of a thermal generation unit is considered as a second order polynomial function (Neglecting valve-point effects) and this is called classical and smooth cost function (refer to (2)).

$$
\mathrm{FC}_{\mathrm{i}}\left(\mathrm{P}_{\mathrm{i}}\right)=\mathrm{a}_{\mathrm{i}} \mathrm{P}_{\mathrm{i}}^{2}+\mathrm{b}_{\mathrm{i}} \mathrm{P}_{\mathrm{i}}+\mathrm{c}_{\mathrm{i}}
$$

where $P_{i}$ is the power of the $i$ th generating unit; $a_{i}, b_{i}, c_{i}$ are the fuel cost coefficients of the $i^{\text {th }}$ generating unit.

\section{B. Non-Smooth Fuel Cost Functions including Valve-point Loading Effects}

The generating units with multi-valve steam turbines exhibit a greater variation in the fuel cost functions. Since the valve point results in the ripples [20-21], a cost function contains higher order nonlinearity. Therefore, the cost function should be modified to consider the valve-point effects. Typically, the valve point results in, as each steam valve starts to open, the ripples like in to take account for the valve-point effects, sinusoidal functions are added to the quadratic cost functions as follows in (3) [22]: 


$$
\begin{aligned}
\mathrm{FC}_{\mathrm{i}}\left(\mathrm{P}_{\mathrm{i}}\right)=\mathrm{a}_{\mathrm{i}} \mathrm{P}_{\mathrm{i}}^{2}+ & \mathrm{b}_{\mathrm{i}} \mathrm{P}_{\mathrm{i}}+\mathrm{c}_{\mathrm{i}} \\
& +\left|\mathrm{e}_{\mathrm{i}} \times \sin \left(\left(\mathrm{f}_{\mathrm{i}} \times\left(\mathrm{P}_{\mathrm{i}, \mathrm{min}}-\mathrm{P}_{\mathrm{i}}\right)\right)\right)\right|
\end{aligned}
$$

where $a_{i}, b_{i}, c_{i}$ are the fuel cost coefficients of the $i^{\text {th }}$ generating unit and $e_{i}, f_{i}$ are the coefficients of generator (i) reflecting valve-point loading effects.

These classical and non-classical models either with smooth or non-smoothed fuel cost functions are subjected to the following equality and inequality constraints:

\section{Equality constraint (Power balance constraint)}

The total power generated must supply the total load demand and the transmission losses, as illustrated in (4).

$$
\sum_{i=1}^{N} P_{i}=P_{\text {Demand }}+P_{\text {Loss }}
$$

where $\mathrm{P}_{\text {Demand }}$ is the total system load demand and $\mathrm{P}_{\text {Loss }}$ is the total line losses. Total line losses can be calculated using (5).

$$
\mathrm{P}_{\text {Loss }}=\sum_{\mathrm{i}=1}^{\mathrm{N}} \sum_{\mathrm{j}=1}^{\mathrm{N}} \mathrm{P}_{\mathrm{i}}^{\mathrm{T}} \mathrm{B}_{\mathrm{ij}} \mathrm{P}_{\mathrm{j}}+\sum_{\mathrm{i}=1}^{\mathrm{N}} \mathrm{P}_{\mathrm{i}} \mathrm{B}_{\mathrm{oi}}+\mathrm{B}_{\mathrm{oo}}
$$

$\mathrm{B}_{\mathrm{ij}}, \mathrm{B}_{\mathrm{oi}}$ and $\mathrm{B}_{\mathrm{oo}}$ are transmission line loss coefficients $\left(\mathrm{P}_{\mathrm{i}}^{\mathrm{T}}\right.$ is vector transpose of all generation plants net $M W, B_{i j}$ is square matrix of same dimension as $\mathrm{P}_{\mathrm{i}}$ and $\mathrm{B}_{\mathrm{oi}}$ is vector of same length as $\mathrm{P}_{\mathrm{i}}$ and $\mathrm{B}_{00}$ is constant).

\section{Inequality constraints (Maximum and minimum limits of power generation)}

Each generator is constrained between its minimum and maximum limits, as shown in (6);

$$
\mathrm{P}_{\mathrm{i}, \min } \leq \mathrm{P}_{\mathrm{i}} \leq \mathrm{P}_{\mathrm{i}, \max } \text { for } \mathrm{i}=1,2, \ldots, \mathrm{N}
$$

Where $\mathrm{P}_{\mathrm{i}}$ is the output power of generator $\mathrm{i}$; $\mathrm{P}_{\mathrm{i}, \text { min }}$ and $P_{i, \text { max }}$ are the min/max power outputs of generator $i$.

\section{OVERVIEW OF GA}

Gas are well-known stochastic methods of global optimization based on the evolution theory of Darwin [4-6]. The GA is a method for solving both constrained and unconstrained optimization problems that is based on natural selection, the process that drives biological evolution. The GA uses three main types of rules at each step to create the next generation from the current population:

- Selection rules select the individuals, called parents that contribute to the population at the next generation.

- Crossover rules combine two parents to form children for the next generation.

- Mutation rules apply random changes to individual parents to form children.

Crossover rate - this rate generally should be high, about 80 95\%. (However some results show that for some problems crossover rate about $60 \%$ is the best).

Mutation rate - on the other side, mutation rate should be very low.

Population size - it may be surprising that a very big population size usually does not improve performance of GA (in meaning of speed of finding solution). A good population size is about 20-30, however sometimes sizes $50-100$ are reported as best. Some research also shows that best population size depends on encoding, on size of encoded string. It means, if you have a chromosome with 32 bits, the population should be say 32, but surely two times more than the best population size for chromosome with 16 bits.

The advantages of GA can be summarized as:

1. Optimizes with continuous or discrete variable.

2. Deals with a large number of variables.

3. Provides a list of optimum variables, not just a single solution.

4. Optimizes variables with extremely complex cost surfaces.

5. May encode the variables so that the optimization is done with the encoded variables and

6. Works with numerically generated data, experimental data, or analytical functions.

\section{A. GA Solution Procedure}

The GA repeatedly modifies a population of individual solutions. Some of the commonly used terminologies in GA are fitness function- which we want to minimize and population- an array of individuals. A generalized procedure for GA is summarized below.

1. The first step is to define the objective function and variables.

2. In the second step the GA parameters are selected and the population is initialized randomly.

3. After initialization the elite randomly and parents are selected based on the fitness value.

4. Once the parents are selected either mutation or crossover is performed form offspring.

5. The offspring is then inserted into the new population.

6. The last step is to check if the optimum solution has been achieved.

\section{B. Terminating the Run of GA Script}

This generational process is repeated until a termination condition has been reached. Common terminating conditions are: set number of iterations, set time reached, a cost that is lower than an acceptable minimum, set number of cost function evaluations, a best solution has not changed after a set number of iterations, or operator termination.

\section{GA AND ELD}

To use GA programming to solve ELD, the following parameters were needed for data input.

1. Number of chromosomes (that comprise a generation).

2. Number of generations.

3. Initial crossover probability (Typically 0.8).

4. Initial mutation probability (0.05\%).

5. Minimal and Maximal power outputs of each unit.

6. B-matrix of line losses.

7. Coefficients of unit fuel cost function including coefficients of valve-point loading.

8. Total load demand.

The objective function and equality, inequality constraints were written in MatLab m-files. GA parameters like population size, initial range, bounds upper and lower limits, selection criteria, crossover function, mutation function, 
stopping criteria and output function were set before running the program. The ranges for the variables were set in the GA and Optimization toolbox of MATLAB version 2010a [23]. The evaluation/fitness function is adopted as illustrated in (7) for evaluating the fitness of each individual in the population.

$$
\text { Minimize: } \mathrm{FC}_{\mathrm{T}}=\sum_{\mathrm{i}=1}^{\mathrm{N}} \mathrm{FC}_{\mathrm{i}}\left(\mathrm{P}_{\mathrm{i}}\right)
$$

\section{CAse Studies And Numerical Simulations}

To verify the feasibility and performance efficiency of applying GA to solve ELD with taking the effect of valve ripples into consideration, several cases were tested and investigated. Among of these, two cases will be presented. The GA based algorithm is applied to solve the six-units with line losses and neglecting effects of valve-loading as Case I. In Case II, the algorithm was applied to forty-unit system with considering valve-point loading effects and neglecting line losses. Simulations were carried out using GA and Optimization toolbox of MatLab ${ }^{2} 7.10$ release 2010a version.

\section{A. Case I: 6-Generators System with line losses (Not include} Valve-loading Effects)

The Fuel cost characteristics in $\$$ / h of the six units and the unit operating min/max (in MW) ranges [19] are given as follows:

$$
\begin{aligned}
& F_{1}=0.0033870 P_{1}^{2}+0.856440 P_{1}+16.817750 \\
& F_{2}=0.002350 P_{2}^{2}+1.0257600 P_{2}+10.029450 \\
& F_{3}=0.0006230 P_{3}^{2}+0.897700 P_{3}+23.333280 \\
& F_{4}=0.0007880 P_{4}^{2}+0.851234 \mathrm{P}_{4}+27.634000 \\
& F_{5}=0.0004690 \mathrm{P}_{5}^{2}+0.807285 \mathrm{P}_{5}+36.856880 \\
& \mathrm{~F}_{6}=0.0003998 \mathrm{P}_{6}^{2}+0.850454 \mathrm{P}_{6}+30.147980
\end{aligned}
$$

\begin{tabular}{c|c|c|c|c|c|c}
\hline \hline Unit & $\mathrm{G}_{1}$ & $\mathrm{G}_{2}$ & $\mathrm{G}_{3}$ & $\mathrm{G}_{4}$ & $\mathrm{G}_{5}$ & $\mathrm{G}_{6}$ \\
\hline $\mathrm{P}_{\min }$ & 10 & 10 & 35 & 35 & 130 & 125 \\
\hline $\mathrm{P}_{\max }$ & 125 & 150 & 225 & 210 & 325 & 315 \\
\hline \hline
\end{tabular}

\begin{tabular}{lccccc}
\multicolumn{5}{l}{ Line Losses Co-efficient $\mathrm{B}_{\mathrm{ij}} \mathrm{X} 10^{-3}$ Matrix } \\
0.140 & 0.017 & 0.015 & 0.019 & 0.026 & 0.022 \\
0.017 & 0.060 & 0.013 & 0.016 & 0.015 & 0.020 \\
0.015 & 0.013 & 0.065 & 0.017 & 0.024 & 0.019 \\
0.019 & 0.016 & 0.017 & 0.071 & 0.030 & 0.025 \\
0.026 & 0.015 & 0.024 & 0.030 & 0.069 & 0.032 \\
0.022 & 0.020 & 0.019 & 0.025 & 0.032 & 0.085
\end{tabular}

Table I shows optimal scheduling of a Six-unit system by GA Method (including transmission losses) with comparison with PSO results reported in [20]. Fig. 1 and Fig. 2 show the convergence graphs for 700 and 800 MW power demands. The results of best fuel costs shown in the graph of Fig. 1 and Fig. 2 are in Indian Rs ( $1 \$=45$ Rs). The elapsed time of GA processing includes the time required for search process and plotting the graph of convergence. Off course without the graph, the time will be less. Anyway, both PSO and GA algorithms need a lot of efforts to tune parameters to work properly and both are using random initial populations.

TABLE I: THE OPTIMAL SCHEDULING OF GENERATORS FOR DEMANDS OF $700 \mathrm{MW}$ AND $800 \mathrm{MW}$.

\begin{tabular}{c|c|c|c|c}
\hline \hline Unit & $\mathbf{P}_{\mathbf{D}}=\mathbf{7 0 0}$ & $\mathbf{P}_{\mathbf{D}}=\mathbf{7 0 0}$ & $\mathbf{P}_{\mathbf{D}}=\mathbf{8 0 0}$ & $\mathbf{P}_{\mathbf{D}}=\mathbf{8 0 0}$ \\
\hline $\mathrm{P}_{1}$ & 16 & 27.30096 & 25 & 32.67373 \\
\hline $\mathrm{P}_{2}$ & 24 & 15.61244 & 12 & 15.81606 \\
\hline $\mathrm{P}_{3}$ & 138 & 120.31087 & 116 & 141.66228 \\
\hline $\mathrm{P}_{4}$ & 116 & 116.77564 & 182 & 131.31169 \\
\hline $\mathrm{P}_{5}$ & 208 & 226.83767 & 287 & 252.37105 \\
\hline $\mathrm{P}_{6}$ & 214 & 212.40501 & 203 & 251.55072 \\
\hline \hline
\end{tabular}

\begin{tabular}{l|c|c|c|c}
\hline \hline $\mathrm{P}_{\text {Loss }}$ & $\mathbf{1 8}$ & $\mathbf{1 9 . 2 4 2 6}$ & $\mathbf{2 6}$ & $\mathbf{2 5 . 3 8 5 5}$ \\
\hline FC $(\$ / \mathrm{h})$ & $\mathbf{8 2 1 . 9 3}$ & $\mathbf{8 2 0 . 4 2}$ & $\mathbf{9 3 5 . 8 7}$ & $\mathbf{9 3 1 . 1 0 6}$ \\
\hline Time $($ Sec.) & 1.2 & $\mathbf{1 . 7 8}$ & 1.6 & 1.74 \\
\hline Method & PSO $[21]$ & GA & PSO [21] & GA \\
\hline \hline
\end{tabular}

FC=Fuel Cost

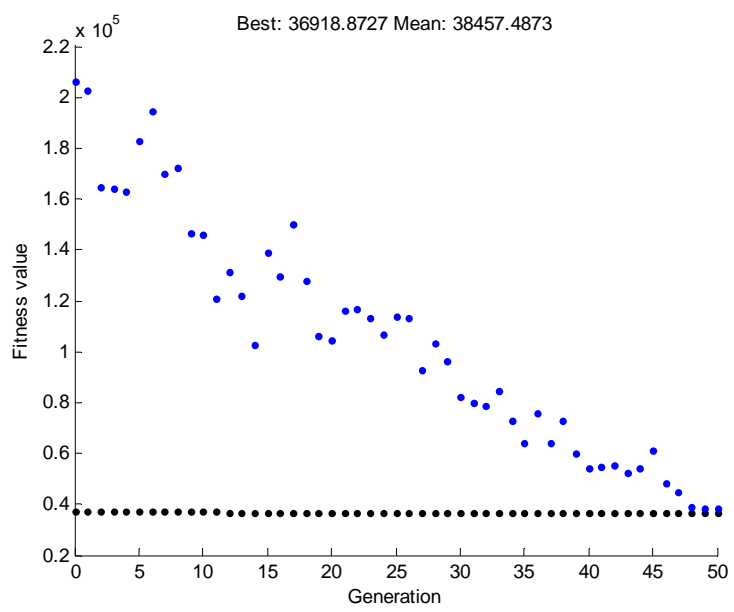

Fig. 1. Convergence graph for 6-units with $P_{D}=700 \mathrm{MW}$

B. Case II: 40-Units with valve-loading effect (neglect losses).

The data of cost coefficients and coefficients reflecting valve-point effects [21-22] is given below in table II. Losses are neglected for sake of comparisons and the case of load demand of 10,500 MW is considered as in [20-21]. Table III shows self-explanatory numerical results obtained with GA in comparisons with PSO approaches in [21-22].

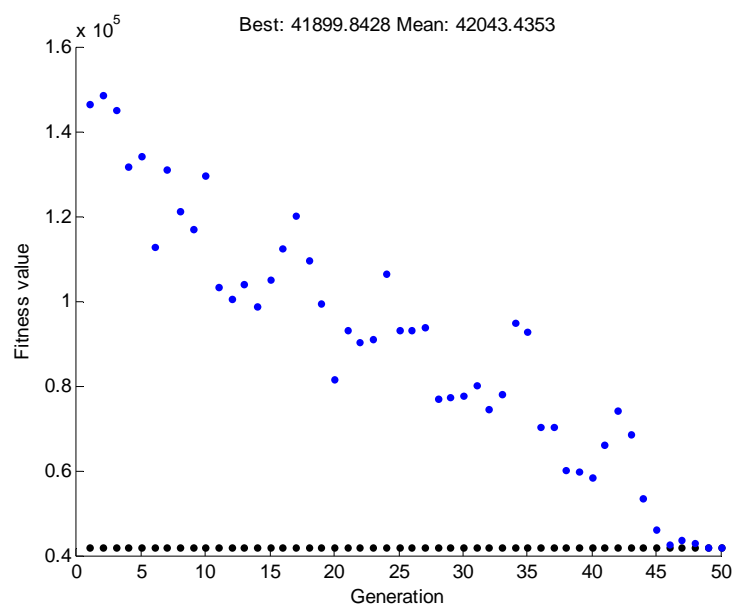

Fig. 2. Convergence graph for 6-units with $P_{D}=800 \mathrm{MW}$

TABLE II: 40-UNITS DATA (NON-SMOOTHING FUEL COST)

\begin{tabular}{c|c|c|c|c|c|c|c}
\hline \hline Unit & $\mathbf{P}_{\mathbf{i}, \min }$ & $\mathbf{P}_{\mathbf{i}, \max }$ & $\mathbf{a}_{\mathbf{i}}$ & $\mathbf{b}_{\mathbf{i}}$ & $\mathbf{c}_{\mathbf{i}}$ & $\mathbf{e}_{\mathbf{i}}$ & $\mathbf{f}_{\mathbf{i}}$ \\
\hline 1 & 36 & 114 & 0.00690 & 6.73 & 94.705 & 100 & 0.084 \\
\hline 2 & 36 & 114 & 0.00690 & 6.73 & 94.705 & 100 & 0.084 \\
\hline 3 & 60 & 120 & 0.02028 & 7.07 & 309.54 & 100 & 0.084 \\
\hline 4 & 80 & 190 & 0.00942 & 8.18 & 369.03 & 150 & 0.063 \\
\hline 5 & 47 & 97 & 0.0114 & 5.35 & 148.89 & 120 & 0.077 \\
\hline 6 & 68 & 140 & 0.01142 & 8.05 & 222.33 & 100 & 0.084 \\
\hline 7 & 110 & 300 & 0.00357 & 8.03 & 287.71 & 200 & 0.042 \\
\hline 8 & 135 & 300 & 0.00492 & 6.99 & 391.98 & 200 & 0.042 \\
\hline 9 & 135 & 300 & 0.00573 & 6.60 & 455.76 & 200 & 0.042 \\
\hline 10 & 130 & 300 & 0.00605 & 12.9 & 722.82 & 200 & 0.042 \\
\hline 12 & 94 & 375 & 0.00515 & 12.9 & 635.20 & 200 & 0.042 \\
\hline 13 & 125 & 500 & 0.00421 & 12.5 & 913.40 & 300 & 0.035 \\
\hline 14 & 125 & 500 & 0.00752 & 8.84 & 1760.4 & 300 & 0.035 \\
\hline 15 & 125 & 500 & 0.00708 & 9.15 & 1728.3 & 300 & 0.035 \\
\hline \multicolumn{7}{|c|}{} & \multicolumn{5}{|c}{}
\end{tabular}




\begin{tabular}{c|c|c|c|c|c|c|c}
\hline 16 & 125 & 500 & 0.00708 & 9.15 & 1728.3 & 300 & 0.035 \\
\hline 17 & 220 & 500 & 0.00313 & 7.97 & 647.85 & 300 & 0.035 \\
\hline 18 & 220 & 500 & 0.00313 & 7.95 & 649.69 & 300 & 0.035 \\
\hline 19 & 242 & 550 & 0.00313 & 7.97 & 647.83 & 300 & 0.035 \\
\hline 20 & 242 & 550 & 0.00313 & 7.97 & 647.81 & 300 & 0.035 \\
\hline 21 & 254 & 550 & 0.00298 & 6.63 & 785.96 & 300 & 0.035 \\
\hline 22 & 254 & 550 & 0.00298 & 6.63 & 785.96 & 300 & 0.035 \\
\hline 23 & 254 & 550 & 0.00284 & 6.66 & 794.53 & 300 & 0.035 \\
\hline 24 & 254 & 550 & 0.00284 & 6.66 & 794.53 & 300 & 0.035 \\
\hline 25 & 254 & 550 & 0.00277 & 7.10 & 801.32 & 300 & 0.035 \\
\hline 26 & 254 & 550 & 0.00277 & 7.10 & 801.32 & 300 & 0.035 \\
\hline 27 & 10 & 150 & 0.52124 & 3.33 & 1055.1 & 120 & 0.077 \\
\hline 28 & 10 & 150 & 0.52124 & 3.33 & 1055.1 & 120 & 0.077 \\
\hline 29 & 10 & 150 & 0.52124 & 3.33 & 1055.1 & 120 & 0.077 \\
\hline 30 & 47 & 97 & 0.01140 & 5.35 & 148.89 & 120 & 0.077 \\
\hline 31 & 60 & 190 & 0.00160 & 6.43 & 222.92 & 150 & 0.063 \\
\hline 32 & 60 & 190 & 0.00160 & 6.43 & 222.92 & 150 & 0.063 \\
\hline 33 & 60 & 190 & 0.00160 & 6.43 & 222.92 & 150 & 0.063 \\
\hline 34 & 90 & 200 & 0.0001 & 8.95 & 107.87 & 200 & 0.042 \\
\hline 35 & 90 & 200 & 0.0001 & 8.62 & 116.58 & 200 & 0.042 \\
\hline 36 & 90 & 200 & 0.0001 & 8.62 & 116.58 & 200 & 0.042 \\
\hline 37 & 25 & 110 & 0.0161 & 5.88 & 307.45 & 80 & 0.098 \\
\hline 38 & 25 & 110 & 0.0161 & 5.88 & 307.45 & 80 & 0.098 \\
\hline 39 & 25 & 110 & 0.0161 & 5.88 & 307.45 & 80 & 0.098 \\
\hline 40 & 242 & 550 & 0.00313 & 7.97 & 647.83 & 300 & 0.035 \\
\hline \hline
\end{tabular}

TABLE III: 40 UNITS OPTIMUM DISPATCH

\begin{tabular}{|c|c|c|c|c|}
\hline \multirow{2}{*}{ Unit } & \multirow{2}{*}{ Ref. [22] } & \multirow{2}{*}{ Ref. [23] } & \multicolumn{2}{|c|}{ Proposed GA } \\
\hline & & & Output & Unit fuel \\
\hline 1 & 114 & 110.8731 & 108.76409 & 918.9593 \\
\hline 2 & 114 & 111.2066 & 114.00000 & 963.00789 \\
\hline 3 & 60 & 97.4 & 117.63920 & 1430.34398 \\
\hline 4 & 190 & 179.7332 & 190.00000 & 2281.39049 \\
\hline 5 & 97 & 87.9256 & 97.00000 & 783.15995 \\
\hline 6 & 140 & 140 & 140.00000 & 1583.69816 \\
\hline 7 & 300 & 259.6023 & 300.00000 & 3045.77548 \\
\hline 8 & 300 & 284.5999 & 300.00000 & 2955.91133 \\
\hline 9 & 290.1619 & 284.6004 & 300.00000 & 2975.59133 \\
\hline 10 & 130 & 130 & 136.56586 & 2598.31611 \\
\hline 11 & 94 & 168.7999 & 94.78717 & 1904.34063 \\
\hline 12 & 94 & 94 & 94.38809 & 1913.60729 \\
\hline 13 & 125 & 214.7598 & 127.91692 & 2581.78318 \\
\hline 14 & 125 & 304.5196 & 311.14543 & 5272.98739 \\
\hline 15 & 394.28 & 394.2794 & 282.76897 & 4910.60871 \\
\hline 16 & 394.28 & 394.2794 & 203.20460 & 3894.29652 \\
\hline 17 & 500 & 489.2794 & 500.00000 & 5466.41285 \\
\hline 18 & 500 & 489.2795 & 500.00000 & 5458.25285 \\
\hline 19 & 550 & 511.2795 & 550.00000 & 6034.26653 \\
\hline 20 & 550 & 511.2794 & 550.00000 & 6034.24653 \\
\hline 21 & 550 & 523.2794 & 550.00000 & 5387.85973 \\
\hline 22 & 550 & 523.2796 & 550.00000 & 5387.85973 \\
\hline 23 & 550 & 523.2795 & 550.00000 & 5370.57973 \\
\hline 24 & 550 & 523.2794 & 550.00000 & 5370.57973 \\
\hline 25 & 550 & 523.2794 & 550.00000 & 5598.19473 \\
\hline 26 & 550 & 523.2794 & 550.00000 & 5598.19473 \\
\hline 27 & 10 & 10 & 14.03671 & 1205.19275 \\
\hline 28 & 10 & 10 & 11.97786 & 1170.08709 \\
\hline 29 & 10 & 10 & 11.30362 & 1159.55107 \\
\hline 30 & 97 & 89.0624 & 97.00000 & 783.15995 \\
\hline 31 & 190 & 190 & 190.00000 & 1523.74843 \\
\hline 32 & 190 & 190 & 190.00000 & 1523.74843 \\
\hline 33 & 190 & 190 & 190.00000 & 1523.74843 \\
\hline 34 & 200 & 200 & 200.00000 & 1917.97937 \\
\hline 35 & 200 & 172.2847 & 200.00000 & 1860.68937 \\
\hline 36 & 200 & 200 & 200.00000 & 1860.68937 \\
\hline 37 & 110 & 110 & 107.50147 & 1136.87092 \\
\hline 38 & 110 & 110 & 110.00000 & 1160.64994 \\
\hline 39 & 110 & 110 & 110.00000 & 1160.64994 \\
\hline 40 & 511.28 & 511.2794 & 550.00000 & 6034.26653 \\
\hline Total & $10,500 \mathrm{MW}$ & $10,500 \mathrm{MW}$ & \multicolumn{2}{|c|}{ 10,500 MW } \\
\hline Total & $124,577.273^{*}$ & $121,432.177^{*}$ & \multicolumn{2}{|c|}{$119,732.25$} \\
\hline
\end{tabular}

It is clear and self-explanatory from table III that the proposed GA has provided better solutions compared with PSO approaches.

Tuned GA Options/Parameters for Case II are Generations: 80, Population size: 500, Crossover rate: 0.8 and Migration rate: 0.1 .

*The author has doubt about the total fuel costs of [21] and [22] and believes that the reported fuel costs [21] and [22] should be as Numerals in bold italic between brackets as shown in last row of table III.

Fig. 3 showed the convergence of the solution obtained by GA. The total of 80 iterations was spent during this process with elapsed time 7.34 seconds.

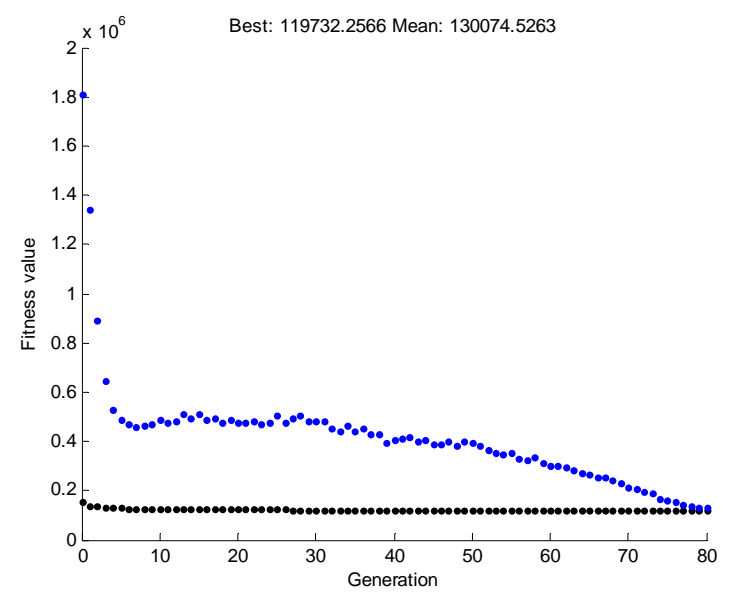

Fig. 3 Solution convergence by GA for 40 units

The best fuel cost results by GA method is much lower than the reported results in [21-22] with smooth convergence as illustrated in Fig. 3.

\section{CONCLUSION}

This paper presents an application of GA approach for solving smooth and non-smooth ELD problem with valve-point ripple effects. GA always found solutions with global minimum or even near to global minimum of total fuel costs. These solutions may be changed from run to run as the GA normally uses an initial population randomly. The algorithm had been applied successfully to ELD with considering the effects of valve-point loading while relaxing all other equality and inequality constraints. Main disadvantage of GA, that lot of efforts were exerted to tune parameters by trial and error for better performance. However, GA is searching with population not like Pattern search that normally uses single initial point. However, GA is can be applied simply after formulating the objectives and constraints. In general, GA can provide good performance for Power System Optimization problems.

\section{REFERENCES}

[1] A.J Wood and B.F. Wollenberg,, Power generation operation and control, John Wiley and Sons, New York, $2^{\text {nd }}$ Edition, 1996.

[2] Hadi Sadaat. Power system analysis. International editions. WCB/McGraw-Hill. 1999.

[3] Jizhong Zhu, Optimization of Power System Operation, ISBN: 978-0-470-29888-6, Copyright (c) 2009 by IEEE.

[4] Saruhan, H., "Genetic Algorithms: An Optimization Technique", Technology, vol. 7, pp. 105-114, 2004. 
[5] K. Y. Lee and M. A. El-Sharkawi (Editors), "Modern Heuristic Optimization Techniques with Applications to Power Systems”, IEEE Power Engineering Society (02TP160), 2002.

[6] R. C. Bansal, "Optimization methods for electric power systems: An overview”, Int. J. Emerging Elect. Power Syst., vol. 2, no. 1, pp. 1-23, 2005.

[7] Feng Gao, "Economic Dispatch Algorithms for Thermal Unit System Involving Combined Cycle Units”, 15th PSCC, Liege, 22-26 August 2005.

[8] Amir Mohammadi, et al, "Online Solving Of Economic Dispatch Problem Using Neural Network Approach And Comparing It With Classical Method”, IEEE-ICET 2006 2nd International Conference on Emerging Technologies Peshawar, Pakistan 13-14 November 2006.

[9] MIKAC, B.-INKRET, R., "Application of a Genetic Algorithm to the Availability-Cost Optimisation of a Transmission Network Topology", Proceedings ICANNGA'97 Third International Conference on Artificial Neural Networks and Genetic Algorithms, Norwich, U.K., Springer Verlag Wien, New York, 1997, pp. 306-310.

[10] D. Attaviriyanupap, H. Kita, E. Tanaka, and J. Hasegawa, “A hybrid EP and SQP for dynamic economic dispatch with nonsmooth incremental fuel cost function”, IEEE Trans. Power Syst., vol. 17, no.2, pp. 411-416, 2002.

[11] N. Sinha, R. Chakrabarti, and P. K. Chattopadhyay, "Evolutionary programming Techniques for economic load dispatch”, IEEE Trans. Evol. Comput, vol. 7, pp. 83-94, Feb. 2003.

[12] S. Khamsawang and S. Jiriwibhakorn, "Solving the Economic Dispatch Problem using Novel Particle Swarm Optimization", International Journal of Electrical, Computer, and Systems Engineering, 2009

[13] Z.L. Gaing, "Particle Swarm Optimization to Solving the Economic Dispatch Considering the Generator Constraints", IEEE Transactions on Power Systems, Vol. 18, No. 3, pp. 1187-1195 August 2003.

[14] Z. L. Gaing, "Constrained dynamic economic dispatch solution using particle swarm optimization", in Proc. IEEE Power Engineering Society General Meeting, pp. 153-158, 2004.

[15] J.B.Park , K.S.Lee, K.Y.Lee, "A Patticle Swarm Optimization for Economic Dispatch with Non-smooth Cost Function”, IEEE Trans. Power Systems, Vol 20 , Feb 2005.

[16] A. Bakirtzis, etal. "Genetic algorithm solution to the economic dispatch problem”, IEE Proc. Generation, Transmission and Distribution, vol. 141, no. 4, pp. 377-382, July 1994.
[17] P.H. Chen and H.C. Chang, "Large scale economic dispatch by genetic algorithm”, IEEE Trans. Power Systems, vol. 10, no. 4, pp. 1919-1926, Nov.1995.

[18] W. Ongsakul, and N. Ruangpayoongsak, "Constrained dynamic economic dispatch by simulated annealing/genetic algorithms", in Proc. 22nd Power Ind. Computer Applications (PICA), Sydney, Australia, 2001, pp. 207-212.

[19] F. BENHAMIDA, "A Hopfield Solution to Economic Dispatch Problem with Transmission Losses", ACTA ELECTROTEHNICA, PP. 314-322, Volume 49, Number 3, 2008,

[20] M. Sudhakaran, et al., "Application of Particle Swarm Optimization for Economic Load Dispatch Problems", The 14th International Conference on Intelligent System Applications to Power Systems, ISAP 2007, PP 668-674.

[21] R.S.Dhivy Apragash, et al., "An Efficient Particle Swarm Optimization for Economic Dispatch Problems with Non-smooth cost functions", WSEAS TRANSACTIONS on POWER SYSTEMS, ISSN: 1790-5060 Issue 4, Volume 3, April 2008, PP 257-266

[22] Jong-Bae Park, et al.,”An Improved PSO for ED with Valve-point Effect", International Journal of Innovations in Energy Systems and Power, Vol. 1, no. 1 (Nov., 2006).

[23] Chen.P.H, and Chang.H.C, "Large-scale economic dispatch by genetic algorithm”, IEEE trans. on Power Systems, Vol.10, N0.4, November, 1995, pp.1919-1926.

[24] http://www.mathworks.com.

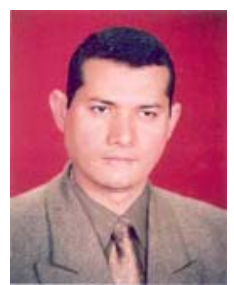

Attia El-Fergany was born in Sharkia, Egypt, on 1971. He graduated from the University of Zagazig. He received M.Sc. and Ph.D. degrees from Zagazig University in 1998 and 2001, respectively. He has been on the Faculty of Engineering as a Lecturer of Electrical Engineering Department. His research interests include power system protection, optimization of power system operations and artificial intelligence applications in power systems. Dr. Atti has been involved in many training sessions for graduate Engineers with different Nationalities and many consultation activities.

Dr. El-Fergany is a senior member of IACSIT and member of IEEE. 